\title{
Fluorescence, Decay Time, and Structural Change of Laser Dye Cresyl Violet in Solution due to Microwave Irradiation at GSM 900/1800 Mobile Phone Frequencies
}

\author{
Fuat Bayrakceken $^{1}$ and Korkut Yegin ${ }^{2}$ \\ ${ }^{1}$ Department of Biomedical Engineering, Yeditepe University, 34755 Istanbul, Turkey \\ ${ }^{2}$ Department of Electrical and Electronics Engineering, Yeditepe University, 34755 Istanbul, Turkey
}

Correspondence should be addressed to Korkut Yegin, kyegin@yeditepe.edu.tr

Received 24 May 2012; Accepted 3 October 2012

Academic Editor: Necdet Aslan

Copyright ( $\odot 2012$ F. Bayrakceken and K. Yegin. This is an open access article distributed under the Creative Commons Attribution License, which permits unrestricted use, distribution, and reproduction in any medium, provided the original work is properly cited.

\begin{abstract}
Microwave irradiation at GSM 900/1800 MHz mobile phone frequencies affects the electronic structure of cresyl violet in solution. These changes are important because laser-dye cresyl violet strongly bonds to DNA- and RNA-rich cell compounds in nerve tissues. The irradiation effects on the electronic structure of cresyl violet and its fluorescence data were all obtained experimentally at room temperature. For most laser dyes, this is not a trivial task because laser dye molecules possess a relatively complex structure. They usually consist of an extended system of conjugated double or aromatic $\pi$-bonds with attached auxochromic (electron donating) groups shifting the absorption band further towards longer wavelength. Because of the intrinsically high degree of conjugation, the vibrational modes of the molecular units couple strongly with each other. We found that the fluorescence quantum yield was increased from $0.54 \pm 0.03$ to $0.75 \pm 0.01$ due to intramolecular energy hopping of cresyl violet in solution which is exposed to microwave irradiation at mobile phone frequencies, and the photonic product cannot be used as a laser dye anymore.
\end{abstract}

\section{Introduction}

Cresyl violet (Figure 1) is often used as a staining agent for studying the changes on DNA- and RNA-rich compounds in tissues. Especially, the effects of microwave radiation on blood-brain barrier permeability heavily rely on cresyl violet based stain analysis, simply because laser dye molecules are often distinguished due to their relatively complex structure in photoluminescence data. However, effects of microwave irradiation on cresyl violet in solution have not been studied in detail, and changes in the absorption band due to microwave irradiation are particularly important for widespread applications of laser dye cresyl violet.

The photophysical and chemical behaviors of cresyl violet are strongly influenced by electrostatic, hydrogenbond, and acid-base interactions in solution, as well as by its hydrophobicity [1]. The rotational relaxation of a solute molecule depends on its environment, especially for the case of ionic and polar solute species embedded in electrolyte solutions [2-5]. In such cases, solute-solvent electrostatic interactions can significantly influence the behavior of the solute. Dramatic changes in the local solvent structure and the solute dynamics occur for ionic solute molecules. Laser dyes are organic compounds that relax radiatively after optical excitation, emitting in the UV-visible or IR range [6]. Today a large variety of luminescent dyes have been optimized for use in circulating liquid lasers. These dyes have a range of practical applications when rigidized in a polymer host. One area that may be revolutionized by the use of organic luminophores in plastics is the flat-screen monitor industry. Organic emitters doped in a polymer layer offer an inexpensive, malleable, and easy-toproduce alternative. Organic dyes are also of interest for sensors, optical amplifiers, and fiber optics. Unfortunately, upon repeated absorption the laser dye molecules begin to photooxidize and cresyl violet becomes an emitter at four different wavelengths. It is stable under ambient conditions with minimal light power saturation even at peak intensities 
<smiles>Cc1cc2c(cc1N(C)C)COC1=CC(=[NH2+])c3ccccc3C1=N2</smiles>

FIGURE 1: Molecular structure of cresyl violet.

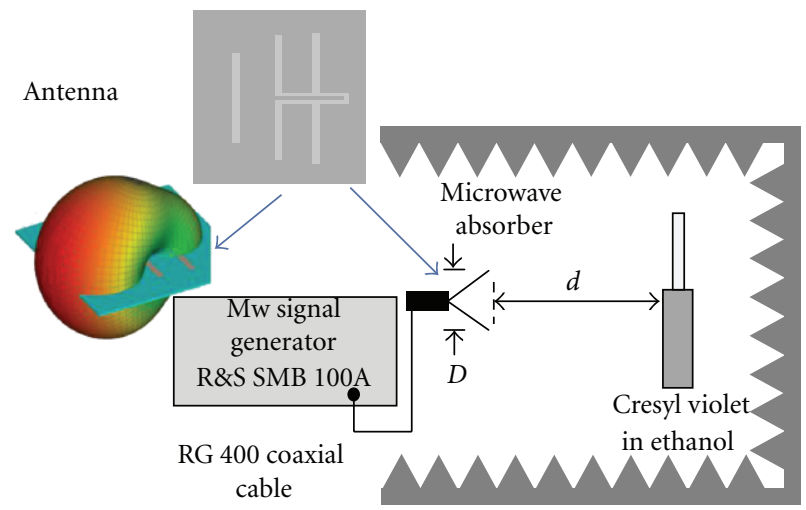

FIGURE 2: microwave irradiation set up, antenna configuration (front and back), and antenna radiation pattern.

as high as $100 \mathrm{MW} / \mathrm{cm}^{2}$ where its molecular weight is $361.74 \mathrm{~g} / \mathrm{moL}$.

Our experiment consisted of irradiating cresyl violet in solution which was doped in thin solid slabs of polymethylmethacrylate with progressively higher intensities of light tuned to an absorption peak of cresyl violet molecule for photobleaching experiments. The calculated wavenumbers of the normal modes are in good agreement with our experimental values. Some of the characteristic vibrational modes of cresyl violet were given [7]. The correlation between effective dipole moments change of the guest molecule and the hole-burning efficiencies of the host matrices illustrates the sensitivity of the dipole moment change as a direct measure of guest-host interactions. The effective dipole moment change of cresyl-violet-perchlorate ranges from 0.14 to 0.59 Debye [8-10].

\section{Materials and Methods}

Cresyl violet, was of Aldrich reagent grade, used as received without any further purification. All other chemicals were also of reagent grade. Cresyl violet, at a concentration of $2 \times 10^{-5} \mathrm{M}$, was in ethanol at room temperature. Ocean Optics spectrophotometer was used for recording absorption spectra. The singlet and triplet transient phenomena were excited with a laser flash photolysis setup consisting of a Q-switched and mode-locked Nd:YAG laser system at $532 \mathrm{~nm}(300 \mathrm{~mJ})$ and at $1064 \mathrm{~nm}(1 \mathrm{~J})$. A kinetic absorption spectrophotometer with nanosecond response (pulsed

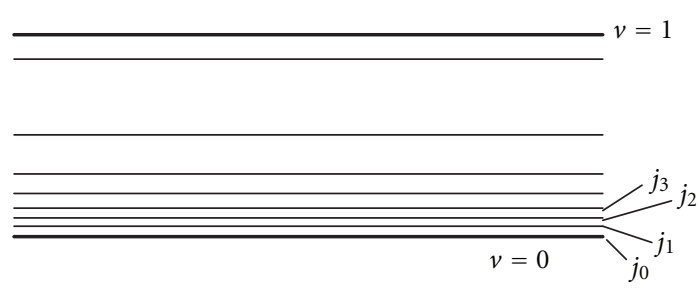

FIGURE 3: Illustration of rotational states as sublevels of $v=0$ state.

with $500 \mathrm{~W}$ Xenon lamp), Bausch and Lomb UV-visible high-intensity monochromator, RCA-4840 photomultiplier tube with output signal terminated into $93 \Omega$ a Tektronix 7912 digitizer, and an LSI-11 microprocessor unit which controlled the experiments and processed the data at the initial stage, were used. The flash photolysis experiments were carried out with oxygen-free nitrogen, degassed in the high vacuum system (vacuum line background pressure was $10^{-7}$ Torr) and contained in $1 \times 0.2 \mathrm{~cm}^{2}$ quartz cells with the absorbed light passing along the $0.2 \mathrm{~cm}$ path length. The exciting laser beam intersected the cell at angles ranging from $20^{\circ}-90^{\circ}$, and the flash duration time was $3.0 \pm 0.1 \mathrm{~ns}$.

The microwave irradiation frequencies for the cresyl violet in ethanol are chosen as $925 \mathrm{MHz}$, center frequency of GSM $900(890-960 \mathrm{MHz})$ and $1940 \mathrm{MHz}$, center frequency of GSM 1800/1900 and 3G frequency band (1710$2170 \mathrm{MHz}$ ). Frequencies are not applied at the same time, but rather half of the target duration with $925 \mathrm{MHz}$ and the other half with $1940 \mathrm{MHz}$. Although cellular phone communication utilizes pulsed transmissions in a time-shared manner, we applied continuous wave (CW) with no signal modulation in our experiments. This deviation from reallife application does not affect current analysis as irradiation effects rather than interference effects are scrutinized. CW power level is set to $30 \mathrm{dBm}(1 \mathrm{~W})$ using Rohde-Schwarz SMB 100A signal generator. An antenna is connected to the signal generator with microwave coaxial cable, RG400A to transmit microwave energy to the solution. A balanced type, three-element (reflector, driver, and director) directional printed dipole Yagi antenna is designed at $1940 \mathrm{MHz}$. The same antenna works well at $925 \mathrm{MHz}$ also with a bit sacrifice on impedance match. Also, the antenna has an integrated quarter-wavelength Marchand balun (balanced to unbalanced) to minimize the coupling between the antenna and the measurement cable and to reduce the loading effects of the sample on the antenna. The antenna geometry and the integrated balun are designed with a $3 \mathrm{D}$ electromagnetic field solver. Although the computed total gain (vertical and horizontal) at its principal axis of the antenna is $5.73 \mathrm{dBi}$ (relative to isotropic source) at $1940 \mathrm{MHz}$, near-field (beyond reactive zone) electric field illumination at the solution is important. The antenna input impedance is matched to that of the signal generator, which is $50 \Omega$. The antenna is built on a low-cost, two-sided FR4 substrate with $1.57 \mathrm{~mm}$ standard thickness and its one-port characteristics are measured with Rohde-Schwarz ZVB20 network analyzer. The shape of the antenna (front and back), 3D far-field pattern, and the 


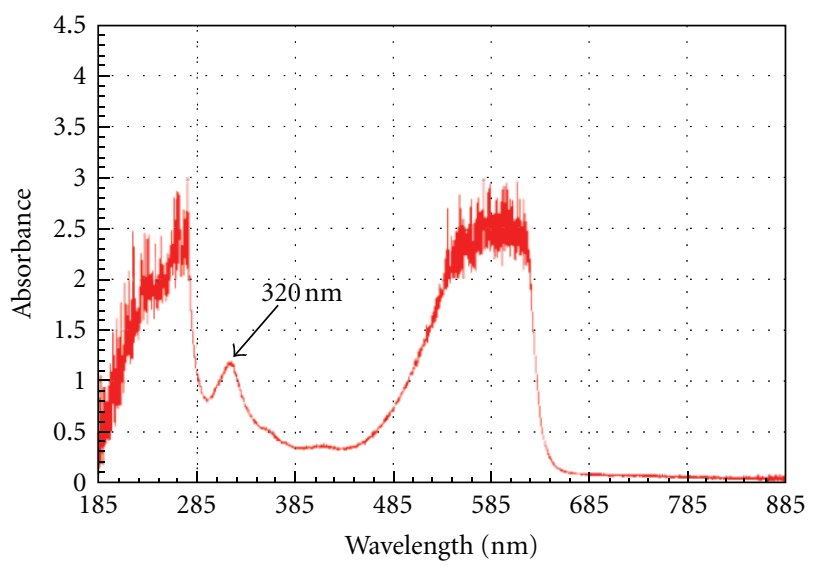

Reference

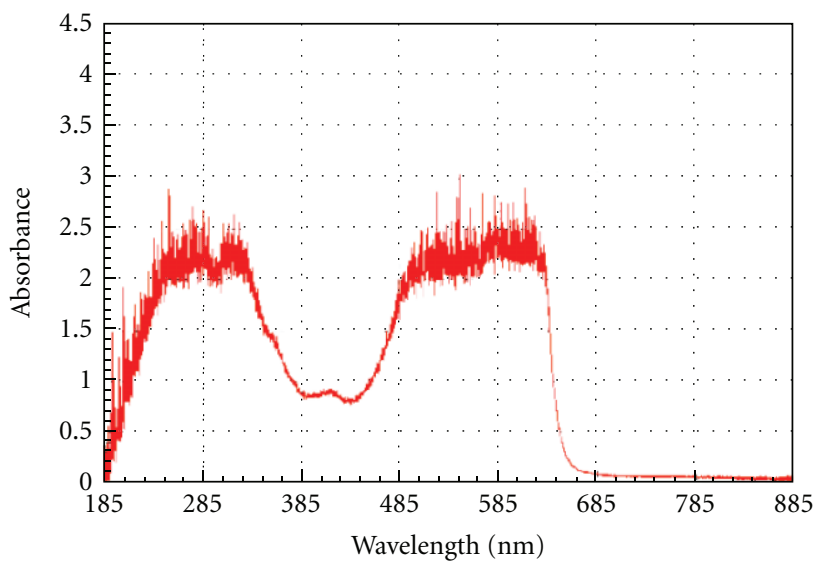

— $1 \mathrm{hr}$ irradiated

(a)

(b)

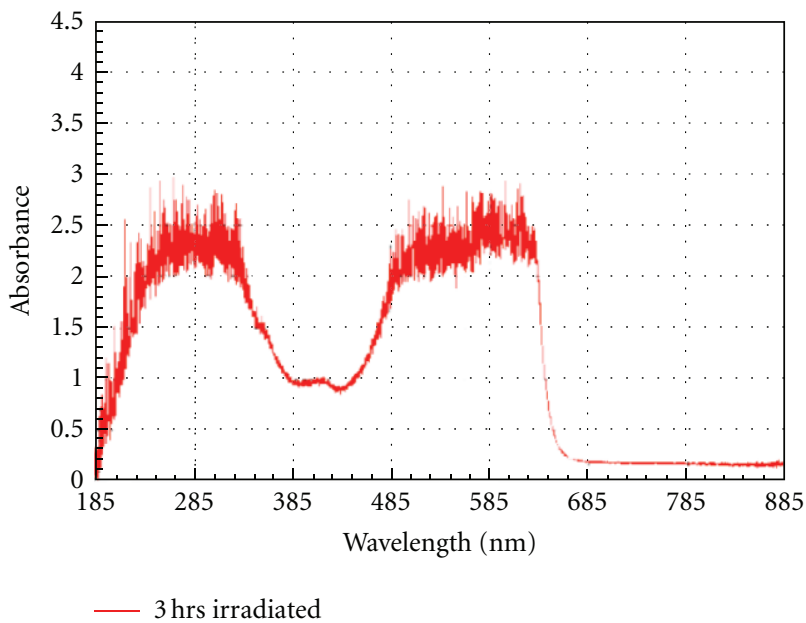

(c)

FIgURE 4: Absorption spectra of cresyl violet in ethanol; (a) reference (non-irradiated), (b) 1 hour irradiated, (c) 3 hours irradiated.

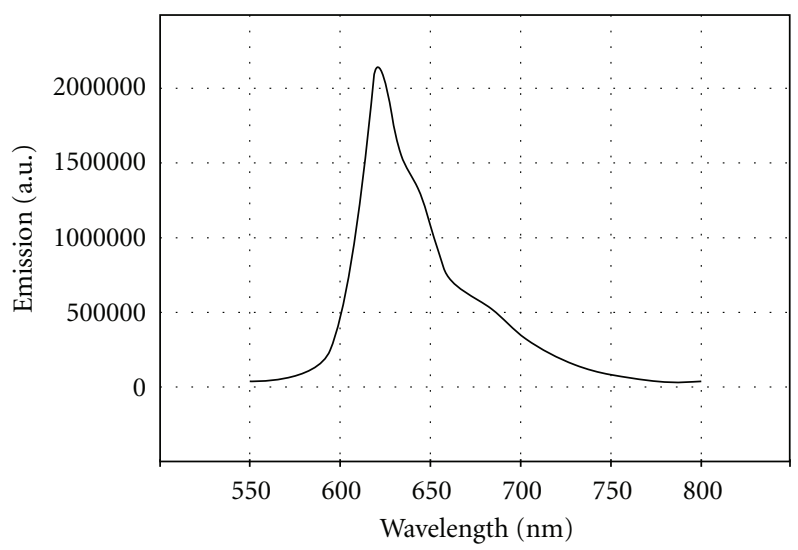

FIgURe 5: Prompt fluorescence spectrum of CV in ethanol.

configuration of the irradiation experiment are illustrated in Figure 2.

In the setup, the sample is placed at distance $d(30 \mathrm{~cm})$ away from the phase center of the antenna. The distance $d$ is adjusted to $2 D^{2} / \lambda>d>k D / 2$ where $k$ and $\lambda$ represent the wavenumber and the wavelength corresponding to $1940 \mathrm{MHz}$ CW frequency, respectively, and $D$ is the maximum antenna dimension. With this distance, the sample remains away from the reactive near field but is at radiative near field (Fresnel region). In Fresnel region, the antenna is not detuned due to the presence of the solution, and most of the transmitted power can be absorbed by the solution. For $925 \mathrm{MHz}$ excitation, the distance $d$ is doubled. With a very crude model, assuming the solution is at far field of the antenna, one can estimate the maximum received power by the solution using Friis equation:

Power Transmitted $P_{t}=30 \mathrm{dBm}(1 \mathrm{~W})$

Power Density at $d, P_{d}=\frac{G P_{t}}{4 \pi d^{2}}$

$$
\text { at } d=30 \mathrm{~cm}, P_{d}=3.29 \mathrm{~W} / \mathrm{m}^{2}
$$

Received power, $P_{r}=P_{d} \sigma=658 \mu \mathrm{W}$, 
assuming $2 \mathrm{~cm}^{2}$ incident area. Therefore, 1 hour of irradiation can deliver $2.37 \mathrm{~J}$ of energy to the solution, if the solution absorbs all the microwave energy without reflecting any energy back due to mismatch at the interface between the solution and air. If ethanol (relative permittivity $\varepsilon_{r}=$ 22.55 [10]) is taken as the dominant dielectric for irradiation experiment, then approximately $57.5 \%$ of the energy under normal incidence of electromagnetic wave is absorbed by the solution, that is, $378 \mu \mathrm{W}$ of $658 \mu \mathrm{W}$ incident power.

\section{Results and Discussion}

The molecular structure of cresyl violet and cresyl violet perchlorate in solution were planar in the ground state and twisted in the first excited state with exception of amino groups. The delocalization degree is smaller than that of the double bonds in the molecule. Its fluorescence quantum yield appears to be independent of the nature of the solvent, except in water. Its fluorescence quantum yield was determined using conventional colorimetry as well as thermal lens spectrometry and found to be $\varphi_{F}=0.54 \pm$ 0.03 . The value for $\varphi_{F}$ was reported to be independent of concentration [9]. Therefore, cresyl violet was used as fluorescence standard for the concentrations of $10^{-2}$ to $10^{-3} \mathrm{M}$. In the present study, we tested the concentrations from $10^{-3}$ to $10^{-7} \mathrm{M}$ and found that due to the intramolecular energy hopping of cresyl violet in solution the fluorescence quantum yield was measured as $0.75 \pm 0.01$ by actinometric measurements. The excitation wavelength was $587 \mathrm{~nm}$, obtained from high-intensity mercury light source. Prompt fluorescence observed at $570-770 \mathrm{~nm}$ band with the peak location at $620 \mathrm{~nm}$. Neither Stokes nor anti-Stokes scattering was observed at room temperature experiments. Unfortunately, our experimental setup does not permit us to carry out the same experiments above room temperature, to carry out kinetic measurements. The determined fluorescence lifetime, which is the reorientation time of cresyl violet in ethanol in the $S_{1}$ state, was $4.60 \pm$ $0.1 \mathrm{nsec}$. The magic-angle fluorescence decay curves fit to single-exponential decays.

The amount of thermal energy transferred to the cresyl violet in ethanol due to microwave irradiation is very low compared to the energy required for the transition of electron to a higher vibrational state. However, at each vibrational energy level $J_{i}$, there exists many fine rotational bands, as illustrated in Figure 3. For symmetric top molecules having rigid bonds, the energy levels can be determined. More complicated relations are required for elastic bonds [11]. If the molecule is asymmetric top, then it is very difficult to derive an expression for rotational energy bands for each vibrational states due to different moments of inertia about the molecular axis. Thus, measurement of absorbance spectrum is the only viable solution, particularly for cresyl violet.

The ethanol solution act to differentiate the stain, causing myelin and other components to lose color whereas perikarya retain the color. Figure 4 shows the absorption spectrum of nonirradiated cresyl violet in ethanol and the absorption spectra of cresyl violet after one and three hours of irradiation by microwave. Due to the irradiation by GSM $900 / 1800 \mathrm{MHz}$ mobile phone frequencies, the absorption band at $320 \mathrm{~nm}$ disappeared and a new molecular cresyl violet structure formed. It is also observed that the main absorption bands are broadened and the spectrum showed that the fine structure of the bands appears. Therefore, cresyl violet molecular structure due to the microwave irradiation is changed. The phononic product cannot be used as a laser dye. The prompt fluorescence spectrum of cresyl violet in ethanol solution is shown in Figure 5.

\section{Acknowledgments}

This research is partly funded by NATO under Grant no. CRG-950982 and Fulbright Advanced Research Program at Massachusetts Institute of Technology, USA. Fluorescence spectrum and decay time measurements were carried out at UNAM (National Nanotechnology Research Center), Bilkent University, Turkey.

\section{References}

[1] S. J. Isak and E. M. Eyring, "Cresyl violet chemistry and photophysics in various solvents and micelles," Journal of Photochemistry and Photobiology A, vol. 64, no. 3, pp. 343-358, 1992.

[2] P. G. Wolynes, "Dynamics of electrolyte solutions," Annual Review of Physical Chemistry, vol. 31, pp. 345-376, 1980.

[3] I. Rips, J. Klafter, and J. Jortner, "Dynamics of ionic solvation," The Journal of Chemical Physics, vol. 88, no. 5, pp. 3246-3252, 1988.

[4] J. Hubbard and L. Onsager, "Dielectric dispersion and dielectric friction in electrolyte solutions. I," The Journal of Chemical Physics, vol. 67, no. 11, pp. 4850-4857, 1977.

[5] N. Balabai, M. G. Kurnikova, R. D. Coalson, and D. $\mathrm{H}$. Waldeck, "Rotational relaxation of ionic molecules in electrolyte solutions. Anisotropy relaxation and molecular dynamics study," Journal of the American Chemical Society, vol. 120, no. 31, pp. 7944-7951, 1998.

[6] F. Bayrakceken, I. S. Karaaslan, and B. Erol, "Structure elucidation of cresyl violet perchlorate in polyvinylbutyral," Spectrochim Acta, vol. 68, pp. 139-142, 2007.

[7] E. Vogel, A. Gbureck, and W. Kiefer, "Vibrational spectroscopic studies on the dyes cresyl violet and coumarin 152," Journal of Molecular Structure, vol. 550-551, pp. 177-190, 2000.

[8] A. Cavus, M. Wright, J. R. Lombardi, and F. Bayrakceken, "Stark hole-burning spectroscopy of cresylviolet perchlorate in amorphous hosts," Spectroscopy Letters, vol. 32, no. 1, pp. 125-137, 1999.

[9] F. Bayrakceken, I. Karaaslan, K. Yegin, B. Barýs, and M. Bilgic, "Photonic and phononic study of rubrene in solution," Asian Journal of Spectroscopy, pp. 119-129, 2010.

[10] A. Jafari, A. Ghanadzadeh, H. Tajalli, M. Yeganeh, and M. Moghadam, "Electronic absorption spectra of cresyl violet acetate in anisotropic and isotropic solvents," Spectrochimica Acta A, vol. 66, no. 3, pp. 717-725, 2007.

[11] C. H. Townes and A. L. Schawlow, Microwave Spectroscopy, Dover, New York, NY, USA, 1975. 


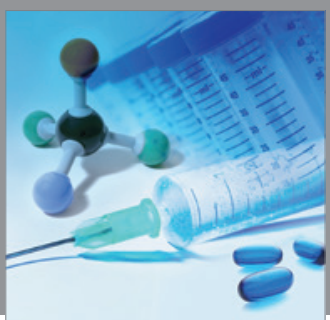

International Journal of

Medicinal Chemistry

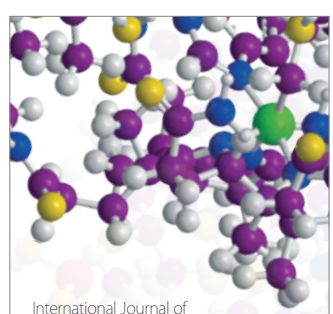

Carbohydrate Chemistry

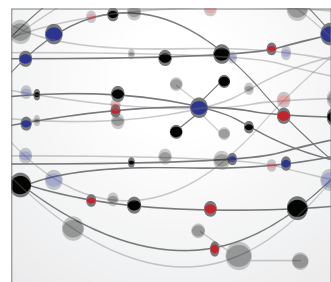

The Scientific World Journal
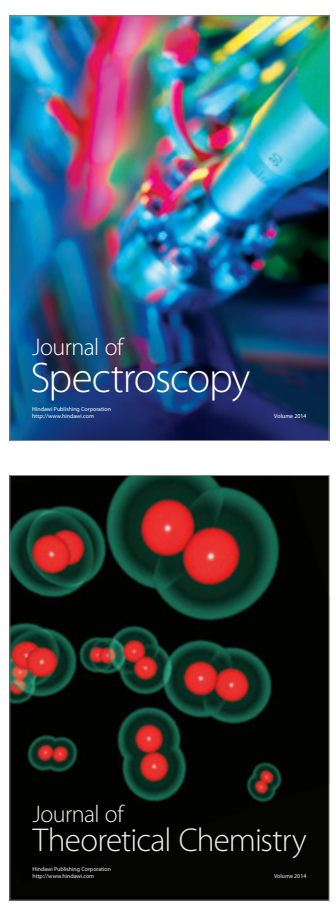
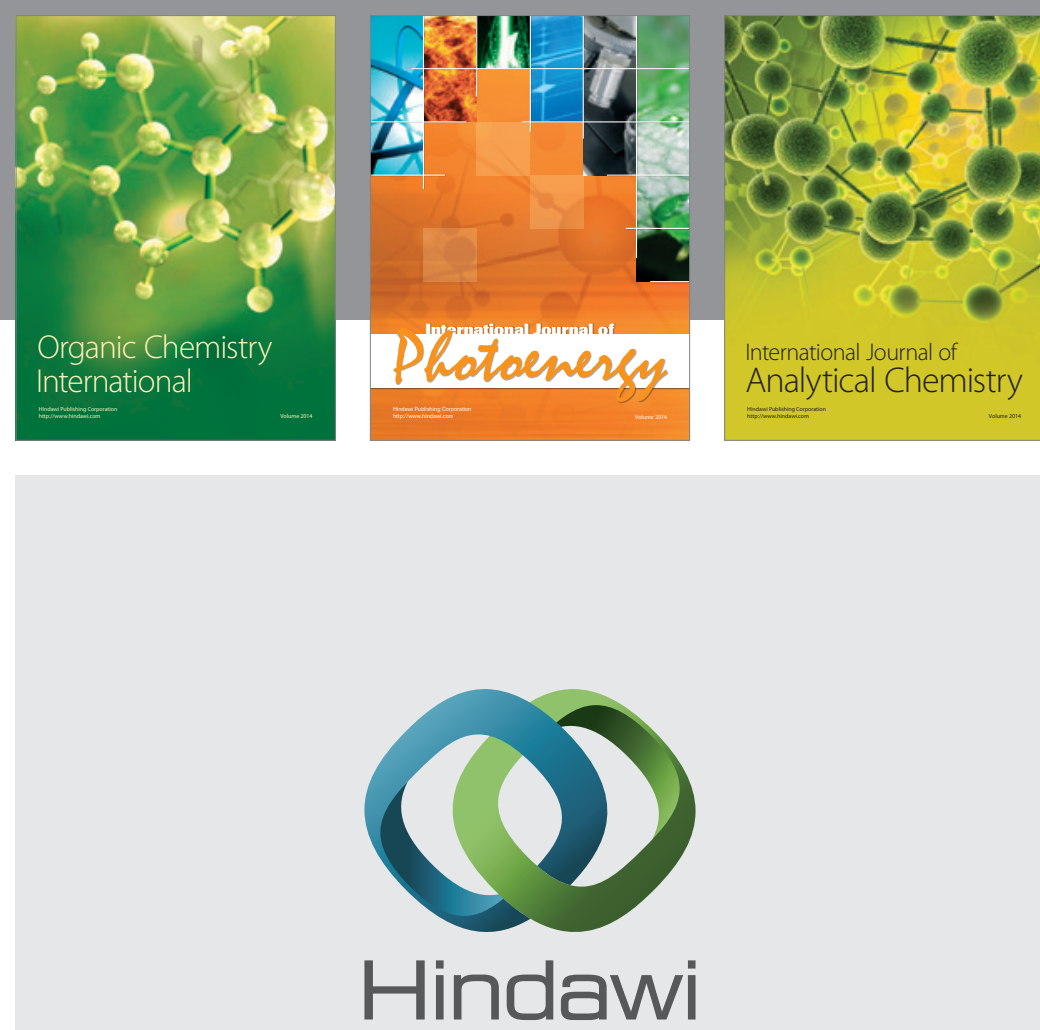

Submit your manuscripts at

http://www.hindawi.com
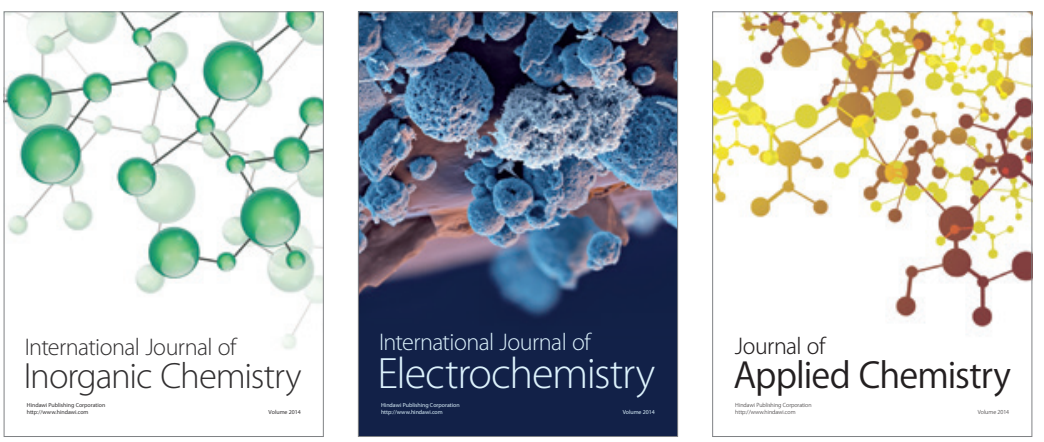

Journal of

Applied Chemistry
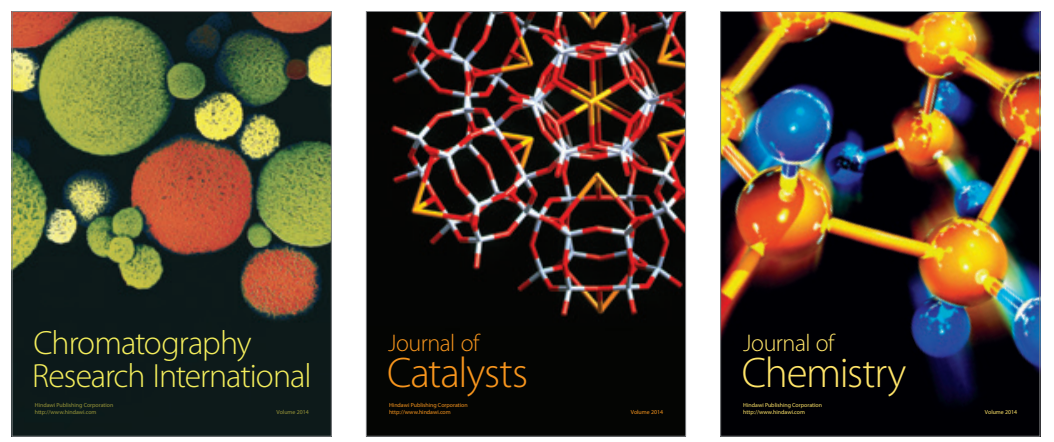
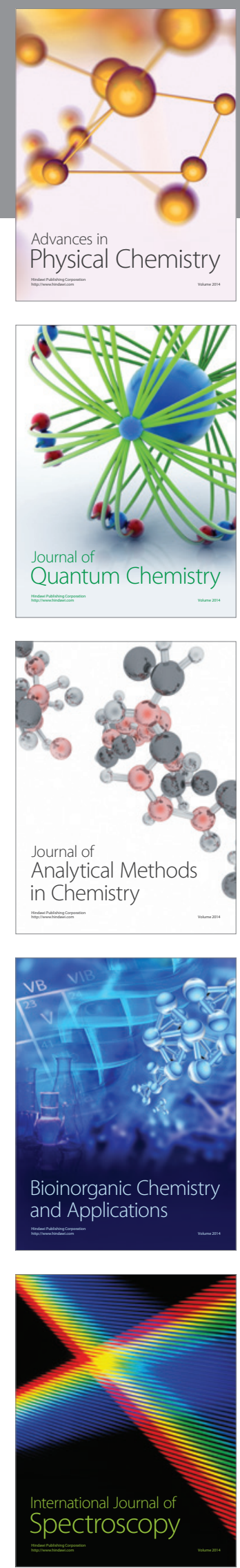